\title{
Walking the Fine Line between Science and Culture
}

\author{
Lee P. Shulman ${ }^{1,2,3}$
}

Published online: 15 July 2015

(c) Springer International Publishing Switzerland 2015

Our abilities to evaluate pregnant women for fetal abnormalities have greatly expanded seemingly overnight as result of advances mostly facilitated by the completion of the Human Genome Project. From the incorporation of cell-free nucleic acid technologies into screening algorithms for fetal chromosomal and select genomic abnormalities to the increasing use of microarrays to detect cytogenetic and genomic abnormalities from amniotic fluid or chorionic villus samples, our capabilities to detect fetal abnormalities have markedly improved over the past 5 years. When combining these improved molecular diagnostics with improving imaging technologies and expertise, we can now accurately detect a wide range of fetal abnormalities, many of which were not detectable a decade ago.

However, before we congratulate ourselves on our accomplishments, it is equally important to recognize that our advancements in diagnostics have not kept pace with

This is a commentary on the paper by Neogi et al. "Indigenous medicine use for sex selection during pregnancy and risk of congenital malformations: a population-based case-control study in Haryana, India,” doi:10.1007/s40264-015-0309-5.

Lee P. Shulman

1ps5@cornell.edu; Lshulman@nm.org

1 Division of Clinical Genetics, Department of Obstetrics and Gynecology, Feinberg School of Medicine of Northwestern University, Chicago, IL, USA

2 Department of Pharmacognosy and Medicine Chemistry, University of Illinois, College of Pharmacy, Chicago, IL, USA

3 Prentice Women's Hospital, 250 E. Superior Street, Room 05-2174, Chicago, IL 60611, USA our understanding of the etiologies of many, if not most fetal abnormalities. Indeed, the long-standing struggle of genetics vs. environment has actually never been more complicated and blurred. It seems that as we learn more about genetic and epigenetic phenomena, the dogma that has supported our beliefs concerning etiology of fetal anomalies becomes weaker and weaker, leaving us with improved abilities to detect these problems but with a far less definitive understanding of what causes these problems, and thus a seemingly increasing inability to offer sage advice to prevent such problems in the future.

Certain conditions such as Mendelian disorders are clearly genetic in nature whereas fetal anomalies resulting from prenatal exposure to teratogenetic agents such as thalidomide and alcohol are mostly mediated by environmental exposure, though the phenotypic expression of such conditions are also impacted by genetic and genomic factors. Indeed, the impact of novel cultural practices, including the ingestion or avoidance of certain foods and medicines before and during pregnancy, can impact fetal development and well-being as is evident in the paper "Indigenous medicine use during pregnancy for sex selection and risk of congenital malformations: a population-based case-control study in Haryana, India" by Sutapa B. Neogi and colleagues, published in this edition of Drug Safety [1].

It is important to accept the notion that culture-specific practices are not automatically bad, harmful, or unnecessary; we tend to consider current approaches to healthcare invariably superior to those from even 20 years ago, let alone those that date back centuries or millennia. However, we should all take a collective deep breath and consider the very recent pharmacotherapeutic advancement in the treatment of methicillin-resistant Staphylococcus 
aureus, a drug-resistant microbe that has become increasingly resistant to more antibiotics over the past several years. What is the recent breakthrough in the treatment of MRSA? A ninth century treatment for eye infections found in Bald's Leechbook, written in Old English and presented as a recipe consisting of garlic and onions, wine, and bile from a cow's stomach brewed in a brass cauldron and left to sit for 9 days before use [2]. I am not sure that this formulation is ready for a phase III trial in preparation for approval by the US Food and Drug Administration or the European Medicines Agency, but I am sure that a successful therapeutic approach for the treatment of MRSA will save thousands of lives.

Therefore, old is not necessarily worse, and the practices described in the paper by Neogi et al. are not restricted to developing nations. In the US and elsewhere, pica refers to cravings during pregnancy that lead to the consumption of nonfood items such as starch and clay that can result in maternal morbidity (e.g., anemia) and, depending on the items consumed, adverse neonatal outcomes [3]. However, in contradistinction to the practices reported by Neogi and colleagues, pica refers to innate cravings by pregnant women that can be influenced by societal or community practices. Whereas the practice of ingesting "botanical interventions" to ensure the delivery of a male child refers to a cultural practice maintained by peer and societal pressures and calls into question not just the ingestion of toxic or teratogenetic substances but the concept of gender as an abnormality.

One can read this paper and applaud the authors' approach to demonstrating the clear teratogenetic impact of the variety of indigenous medicines. In addition, one must also applaud the authors for exposing a practice that is not only biologically absurd, insofar as sex determination occurs at conception and cannot be altered after conception, but also that it continues to this day. Furthermore, that continuation bespeaks an ongoing and even more nefarious issue; specifically, that in Haryana, India, measures must be taken, regardless of their cost, to increase the likelihood of a male child. While not specifically stated, this practice continues because a male child has far more "value" than a female child in Haryana, India.

Many of us look at ritual and cultural practices as quaint, arcane, or even ridiculous, many of which represent an ancient ritual or response to natural phenomena or humanmade events that were not entirely understood by our ancestors. From ritual tribal dances at solar eclipses to the preparation of ceremonial meals, maintaining such practices despite the lack of the original context for their performance serves to honor the past and bring together communities. Some may look at such practices as foreign and ethnocentric, especially when performed outside of the communities in which they were developed. However, acceptance of such practices and gaining an understanding of their origins and meanings is an important part of integrating people of different beliefs and customs into society, something that most communities in the developed world are quickly learning.

However, sometimes such customs, though centuries old, serve to harm the community and alienate people within and without society. The concept of gender as an anomaly is one that requires immediate intervention and termination. Both the overarching desire to reduce female births and the biological absurdity of postconception sex selection require education of the communities in which these practices still occur as well as interventions, both legal and educational, to immediately curtail and eliminate such appalling practices. There are times that sound scientific inquiry uncovers nonscientific issues that require our attention, in addition to the central issues identified by the clinical investigation. This is the case in the work by Neogi and colleagues. Our commitment to improving healthcare must, at times, take us from our "comfort zone" to secure optimal clinical outcomes. Ignoring the issue of gender discrimination in the study published in this edition of Drug Safety in a sense negates the impact of this paper; specifically, that prejudicial and dangerous practices meant to exact an inappropriate outcome need to be stopped not just for the collateral harm exacted on mothers and fetuses but also for the harmful customs that require change regardless of how long they have been practiced or why they were initiated in the first place. While we must all be cognizant of respecting the culture and beliefs of people who may look different than us and who have different views of the world, these differences should not be a barrier to correcting practices that are wrong regardless of the community or location. Human rights should always take precedence over cultural norms and practices. This paper has brought to light a common practice that is ineffective and damaging to exposed fetuses regardless of gender and a practice based in ignorance that views an entire gender at a lower level of existence for no reason other than gender. Commendations to the authors notwithstanding, we all must work to eliminate these practices to support equality and human rights as well as to improve the health and wellbeing of society at large.

\section{Compliance with Ethical Standards}

Funding No sources of funding were used to assist in the preparation of this commentary.

Conflict of interest Lee P. Shulman has no conflicts of interest that are directly relevant to the content of this commentary. 


\section{References}

1. Neogi SB, Negandhi PH, Sandhu N, et al. Indigenous medicine use for sex selection during pregnancy and risk of congenital malformations: a population-based case-control study in Haryana, India. Drug Saf. doi:10.1007/s40264-015-0309-5 (Epub ahead of print).
2. Mahoney MV. Ancient recipe may burst MRSA bubble. Pharmacy Times. 2015. www.pharmacytimes.com. Accessed 22 June 2015.

3. Hamilton S, Rothenberg SJ, Khan FJ, et al. Neonatal lead poisoning from maternal pica behavior during pregnancy. J Natl Med Assoc. 2001;93:317-9. 